\title{
REGULARITY OF A PARABOLIC EQUATION SOLUTION IN A NONSMOOTH AND UNBOUNDED DOMAIN \\ BOUBAKER-KHALED SADALLAH
}

(Received 27 January 2006; accepted 1 August 2006)

Communicated by A. J. Pryde

Abstract

This work is concerned with the problem

$$
\left\{\begin{array}{l}
\partial_{t} u-c(t) \partial_{x}^{2} u=f \\
u_{\mid \partial D \backslash \Gamma_{T}}=0
\end{array}\right.
$$

posed in the domain

$$
D=\left\{(t, x) \in \mathbb{R}^{2} \mid 0<t<T, \varphi_{1}(t)<x<\varphi_{2}(t)\right\},
$$

which is not necessary rectangular, and with

$$
\Gamma_{T}=\left\{(T, x) \mid \varphi_{1}(T)<x<\varphi_{2}(T)\right\} .
$$

Our goal is to find some conditions on the coefficient $c$ and the functions $\left(\varphi_{i}\right)_{i=1,2}$ such that the solution of this problem belongs to the Sobolev space

$$
H^{1,2}(D)=\left\{u \in L^{2}(D) \mid \partial_{t} u \in L^{2}(D), \partial_{x} u \in L^{2}(D), \partial_{x}^{2} u \in L^{2}(D)\right\} .
$$

2000 Mathematics subject classification: primary 35K05, 35K20; secondary 35K.

Keywords and phrases: parabolic, heat equation, nonsmooth domain, sectorial.

\section{Introduction}

In the domain

$$
D=\left\{(t, x) \in \mathbb{R}^{2} \mid 0<t<T, \varphi_{1}(t)<x<\varphi_{2}(t)\right\},
$$

we consider the problem

$$
\left\{\begin{array}{l}
\partial_{t} u-c(t) \partial_{x}^{2} u=f \\
u_{\mid \partial D \backslash \Gamma_{T}}=0
\end{array}\right.
$$

where:

(C) 2008 Australian Mathematical Society 1446-7887/08 \$A2.00+0.00 
(i) $\Gamma_{T}=\left\{(T, x) \mid \varphi_{1}(T)<x<\varphi_{2}(T)\right\}$;

(ii) $c$ is a positive coefficient depending on time;

(iii) $\left(\varphi_{i}\right)_{i=1,2}$ and $c$ are differentiable functions on $] 0, T$ [ satisfying some assumptions to be made precise later on.

The second member $f$ of the equation will be taken in the Lebesgue space $L^{2}(D)$. We look for a solution $u$ of problem $\left(P_{0}\right)$ in the anisotropic Sobolev space

$$
H^{1,2}(D)=\left\{u \in L^{2}(D): \partial_{t} u \in L^{2}(D), \partial_{x} u \in L^{2}(D), \partial_{x}^{2} u \in L^{2}(D)\right\} .
$$

The study of this kind of problems when the coefficient $c$ is constant and $T<+\infty$ has been treated in [19]. In [13], the authors investigated the case when

$$
\left\{\begin{array}{l}
f \text { is in a non-Hilbertian Lebesgue space } \mathrm{L}^{p}(\mathrm{D}) \\
c=1 \\
T<+\infty \\
\varphi_{1}=0 \text { and } \varphi_{2}(t)=t^{\alpha}
\end{array}\right.
$$

they found some conditions on the exponents $\alpha$ and $p$ assuring the optimal regularity of the solution of problem $\left(P_{0}\right)$. It is possible to consider similar questions with some other operators (see, for example, [11, 12]).

Observe that the case where the domain $D$ is cylindrical and $T<+\infty$ is known, for example, in [15] or [1] when the coefficient $c$ is not regular.

During the last decades numerous authors have been interested in the study of many problems posed in bad domains. Among these we can cite [2, 3, 5-11, 16$18,20]$. For bibliographical references see, for example, those of books by [4-7] and the references therein.

In this paper we are interested in particular in the case $T=+\infty, \varphi_{1}(0)=\varphi_{2}(0)$ and $c$ depends on the time. Our main result shows that, thanks to some assumptions on the functions $\left(\varphi_{i}\right)_{i=1,2}$ and $c$, problem $\left(P_{0}\right)$ has a (unique) solution $u$ with optimal regularity, that is $u \in H^{1,2}(D)$ when

$$
D=\left\{(t, x) \in \mathbb{R}^{2} \mid 0<t<+\infty, \varphi_{1}(t)<x<\varphi_{2}(t)\right\},
$$

and $\varphi_{1}(0)=\varphi_{2}(0)$. The proof of this result will be undertaken in four steps:

(1) case of a bounded domain which can be transformed into a rectangle;

(2) case of an unbounded domain which can be transformed into a half strip;

(3) case of a bounded triangular domain;

(4) case of a sectorial domain.

\section{The case of a bounded domain which can be transformed into a rectangle}

Let us consider the problem

$$
\left\{\begin{array}{l}
\partial_{t} u-c(t) \partial_{x}^{2} u=f \in L^{2}\left(D_{1}\right) \\
u_{\mid \partial D_{1} \backslash \Gamma_{T}}=0
\end{array}\right.
$$


where

$$
D_{1}=\left\{(t, x) \in \mathbb{R}^{2} \mid 0<t<T, \varphi_{1}(t)<x<\varphi_{2}(t)\right\},
$$

with the following hypotheses on the functions $\left(\varphi_{i}\right)_{i=1,2}$ and $c$ :

(i) $\quad\left\{\left(\varphi_{i}\right)_{i=1,2}\right.$ and $c$ are continuous functions on $[0, T]$, differentiable on $] 0, T[$; the derivatives $\left(\varphi_{i}^{\prime}\right)_{i=1,2}$ are uniformly bounded;

(ii) there exist two constants $\alpha_{i}>0, i=1,2$, such that $\alpha_{1} \geq c(t) \geq \alpha_{2}$, for all $t \in$ $[0, T]$

(iii) $\varphi_{1}(t)<\varphi_{2}(t)$, for all $t \in[0, T]$;

(iv) $T<+\infty$.

Let $\left(H_{1}\right)$ denote these conditions.

The change of variables $(t, x)$ to $\left(t,\left(x-\varphi_{1}(t)\right) /\left(\varphi_{2}(t)-\varphi_{1}(t)\right)\right)$ transforms $D_{1}$ into $R=] 0, T[\times] 0,1\left[\right.$ and problem $\left(P_{1}\right)$ becomes

$$
\left\{\begin{array}{l}
\partial_{t} u+a(t, x) \partial_{x} u-b(t) \partial_{x}^{2} u=f \in L^{2}(R) \\
u_{\mid \partial R \backslash\{T\} \times] 0,1[}=0,
\end{array}\right.
$$

where

$$
a(t, x)=-\frac{x\left(\varphi_{2}^{\prime}(t)-\varphi_{1}^{\prime}(t)\right)+\varphi_{1}^{\prime}(t)}{\varphi_{2}(t)-\varphi_{1}(t)},
$$

and

$$
b(t)=\frac{c(t)}{\left(\varphi_{2}(t)-\varphi_{1}(t)\right)^{2}} .
$$

Observe that, thanks to hypothesis $\left(H_{1}\right)$, the coefficient $a$ is bounded. So the operator $a(t, x) \partial_{x}: H^{1,2}(R) \rightarrow L^{2}(R)$ is compact. Hence, it is sufficient to study the following problem

$$
\left\{\begin{array}{l}
\partial_{t} u-b(t) \partial_{x}^{2} u=f \in L^{2}(R) \\
u_{\mid \partial R \backslash\{T\} \times] 0,1[}=0 .
\end{array}\right.
$$

It is clear that problem $\left(P_{1}^{\prime \prime}\right)$ admits a (unique) solution $u \in H^{1,2}(R)$ because the coefficient $b$ satisfies the 'uniform parabolicity' condition (see, for example, [1]). On other hand, it is easy to verify that the change of variables $(t, x)$ to $\left(t,\left(x-\varphi_{1}(t)\right) /\left(\varphi_{2}(t)-\varphi_{1}(t)\right)\right)$ conserves the spaces $L^{2}$ and $H^{1,2}$. Consequently, we have the following theorem.

THEOREM 1. If hypothesis $\left(H_{1}\right)$ is satisfied, problem $\left(P_{1}\right)$ admits a (unique) solution $u \in H^{1,2}\left(D_{1}\right)$ in $D_{1}$.

The uniqueness of the solution may be obtained by developing the scalar product $\left(\partial_{t} u-c(t) \partial_{x}^{2} u, u\right)_{L^{2}\left(D_{1}\right)}$. Indeed, we prove that the condition $\partial_{t} u-c(t) \partial_{x}^{2} u=0$ implies $\partial_{x} u=0$. Thus, $\partial_{x}^{2} u=0$. However, $\partial_{t} u-c(t) \partial_{x}^{2} u=0$ leads to $\partial_{t} u=0$. So $u$ is constant and the boundary conditions give $u=0$. 


\section{The case of an unbounded domain which can be transformed into a half strip}

Now, let us consider the problem

$$
\left\{\begin{array}{l}
\partial_{t} u-c(t) \partial_{x}^{2} u=f \in L^{2}\left(D_{2}\right) \\
u_{\mid \partial D_{2}}=0
\end{array}\right.
$$

where

$$
D_{2}=\left\{(t, x) \in \mathbb{R}^{2} \mid 0<t<+\infty, \varphi_{1}(t)<x<\varphi_{2}(t)\right\},
$$

and let $\left(H_{2}\right)$ denote the following conditions on the functions $\left(\varphi_{i}\right)_{i=1,2}$ and $c$ :

(i) $\left\{\begin{array}{l}\left(\varphi_{i}\right)_{i=1,2} \text { and } c \text { are continuous functions on }[0,+\infty[\text {, differentiable on } \\ ] 0,+\infty\left[\text { the derivatives }\left(\varphi_{i}\right)_{i=1,2} \text { are uniformly bounded; }\right.\end{array}\right.$

(ii) there exist $\alpha_{i}>0, i=1,2$ such that $\alpha_{1} \geq c(t) \geq \alpha_{2}>0$, for all $t \in[0,+\infty[$;

(iii) $\varphi_{2}-\varphi_{1}$ is increasing in a neighborhood of $+\infty$; or:

there exists $M>0$ such that $\left|\varphi_{1}^{\prime}(t)-\varphi_{2}^{\prime}(t)\right|\left(\varphi_{2}(t)-\varphi_{1}(t)\right) \leq M . c(t)$;

(iv) $\varphi_{1}(0)<\varphi_{2}(0)$.

The change of variables indicated in the previous section transforms $D_{2}$ into the half strip $B=] 0,+\infty[\times] 0,1\left[\right.$. So problem $\left(P_{2}\right)$ can be written as follows

$$
\left\{\begin{array}{l}
\partial_{t} u+a(t, x) \partial_{x} u-b(t) \partial_{x}^{2} u=f \in L^{2}(B) \\
u_{\mid \partial B}=0
\end{array}\right.
$$

keeping in mind that the coefficients $a$ and $b$ are those defined in Section 2. Let $f_{n}$ be the restriction $f_{\mid] 0, n[\times] 0,1[}$ for all $n \in \mathbb{N}$. Then Theorem 1 shows that for all $n \in \mathbb{N}$, there exists a function $u_{n} \in H^{1,2}\left(B_{n}\right)$ which solves the problem

$$
\left\{\begin{array}{l}
\partial_{t} u_{n}+a(t, x) \partial_{x} u_{n}-b(t) \partial_{x}^{2} u_{n}=f_{n} \in L^{2}\left(B_{n}\right), \\
u_{\left.n \mid \partial B_{n} \backslash\{n\} \times\right] 0,1[}=0,
\end{array}\right.
$$

where $\left.B_{n}=\right] 0, n[\times] 0,1[$.

LEMMA 1. There exists a constant $K$ independent of $n$ such that

$$
\left\|u_{n}\right\|_{L^{2}\left(B_{n}\right)} \leq\left\|\partial_{x} u_{n}\right\|_{L^{2}\left(B_{n}\right)} \leq K\|f\|_{L^{2}(B)} .
$$

PROOF. The Poincaré inequality gives $\left\|u_{n}\right\|_{L^{2}\left(B_{n}\right)} \leq\left\|\partial_{x} u_{n}\right\|_{L^{2}\left(B_{n}\right)}$. Moreover, by developing the scalar product $\left(\partial_{t} u_{n}+a(t, x) \partial_{x} u_{n}-b(t) \partial_{x}^{2} u_{n}, u_{n}\right)$ in $L^{2}\left(B_{n}\right)$ and using condition (iii) in $\left(H_{2}\right)$ we obtain

$$
\begin{aligned}
\left(f_{n}, u_{n}\right) & =\int_{B_{n}} u_{n} \partial_{t} u_{n} d t d x+\int_{B_{n}} a(t, x) u_{n} \partial_{x} u_{n} d t d x-\int_{B_{n}} b(t) u_{n} \partial_{x}^{2} u_{n} d t d x \\
& =\frac{1}{2} \int_{B_{n}} \frac{\varphi_{1}^{\prime}(t)-\varphi_{2}^{\prime}(t)}{\varphi_{1}(t)-\varphi_{2}(t)} u_{n}^{2}(t, x) d t d x+\int_{B_{n}} b(t)\left(\partial_{x} u_{n}\right)^{2} d t d x \\
& \geq \int_{B_{n}} b(t)\left(\partial_{x} u_{n}\right)^{2} d t d x \geq \alpha^{2}\left\|\partial_{x} u_{n}\right\|_{L^{2}\left(B_{n}\right)}^{2} .
\end{aligned}
$$


Hence, for all $\epsilon>0$,

$$
\begin{aligned}
\left\|\partial_{x} u_{n}\right\|_{L^{2}\left(B_{n}\right)}^{2} & \leq \frac{1}{\alpha^{2}}\left\|u_{n}\right\|_{L^{2}\left(B_{n}\right)}\left\|f_{n}\right\|_{L^{2}\left(B_{n}\right)} \\
& \leq \frac{1}{\alpha^{2} \epsilon}\|f\|_{L^{2}(B)}+\frac{\epsilon}{\alpha^{2}}\left\|u_{n}\right\|_{L^{2}\left(B_{n}\right)} .
\end{aligned}
$$

By choosing $\epsilon$ small enough, we prove the existence of a constant $K$ such that $\left\|\partial_{x} u_{n}\right\|_{L^{2}\left(B_{n}\right)} \leq K\|f\|_{L^{2}(B)}$.

REMARK 1. Similar computations show that the same result holds true when we substitute the condition that $\varphi_{2}-\varphi_{1}$ increases in a neighborhood of $+\infty$ by the following

$$
\left|\varphi_{1}^{\prime}(t)-\varphi_{2}^{\prime}(t)\right|\left(\varphi_{2}(t)-\varphi_{1}(t)\right) \leq M c(t) .
$$

PROPOSITION 1. There exists a constant $K$ independent of $n$ such that

$$
\left\|u_{n}\right\|_{H^{1,2}\left(B_{n}\right)} \leq K\|f\|_{L^{2}(B)} .
$$

PROOF. We have

$$
\begin{aligned}
\left\|f_{n}\right\|_{L^{2}(B)}^{2}= & \left(\partial_{t} u_{n}+a(t, x) \partial_{x} u_{n}-b(t) \partial_{x}^{2} u_{n}, \partial_{t} u_{n}+a(t, x) \partial_{x} u_{n}-b(t) \partial_{x}^{2} u_{n}\right)_{L^{2}\left(B_{n}\right)} \\
= & \left\|\partial_{t} u_{n}\right\|_{L^{2}\left(B_{n}\right)}^{2}+\left\|a \cdot \partial_{x} u_{n}\right\|_{L^{2}\left(B_{n}\right)}^{2}+\left\|b . \partial_{x}^{2} u_{n}\right\|_{L^{2}\left(B_{n}\right)}^{2} \\
& +2 \int_{B_{n}} a \partial_{t} u_{n} . \partial_{x} u_{n} d t d x-2 \int_{B_{n}} a b \partial_{x} u_{n} . \partial_{x}^{2} u_{n} d t d x \\
& -2 \int_{B_{n}} b \partial_{t} u_{n} . \partial_{x}^{2} u_{n} d t d x
\end{aligned}
$$

Observe that the conditions (i), (iii) and (iv) of $\left(\mathrm{H}_{2}\right)$ show that the coefficients $a$ and $b$ are bounded. So, thanks to Lemma 1 , for all $\epsilon>0$ we obtain

$$
\begin{aligned}
\left\|\partial_{t} u_{n}\right\|_{L^{2}\left(B_{n}\right)}^{2}+\left\|b . \partial_{x}^{2} u_{n}\right\|_{L^{2}\left(B_{n}\right)}^{2}-2 \int_{B_{n}} b \partial_{t} u_{n} . \partial_{x}^{2} u_{n} d t d x \\
\leq\|f\|_{L^{2}(B)}^{2}+\left\|a \cdot \partial_{x} u_{n}\right\|_{L^{2}\left(B_{n}\right)}^{2}+2\left\|\partial_{t} u_{n}\right\|_{L^{2}\left(B_{n}\right)}\left\|a . \partial_{x} u_{n}\right\|_{L^{2}\left(B_{n}\right)} \\
\quad+2\left\|\partial_{x}^{2} u_{n}\right\|_{L^{2}\left(B_{n}\right)}\left\|a b . \partial_{x} u_{n}\right\|_{L^{2}\left(B_{n}\right)} \\
\quad \leq\|f\|_{L^{2}(B)}^{2}+K_{1}\left(1+\frac{2}{\epsilon}\right)\left\|\partial_{x} u_{n}\right\|_{L^{2}\left(B_{n}\right)}^{2}+\epsilon\left\|\partial_{t} u_{n}\right\|_{L^{2}\left(B_{n}\right)}^{2}+\epsilon\left\|\partial_{x}^{2} u_{n}\right\|_{L^{2}\left(B_{n}\right)}^{2} \\
\leq K_{\epsilon}\|f\|_{L^{2}(B)}^{2}+\epsilon\left\|\partial_{t} u_{n}\right\|_{L^{2}\left(B_{n}\right)}^{2}+\epsilon\left\|\partial_{x}^{2} u_{n}\right\|_{L^{2}\left(B_{n}\right)}^{2},
\end{aligned}
$$

where $K_{1}$ and $K_{\epsilon}$ are constants independent of $n$. Consequently,

$$
(1-\epsilon)\left(\left\|\partial_{t} u_{n}\right\|_{L^{2}\left(B_{n}\right)}^{2}+\left\|b . \partial_{x}^{2} u_{n}\right\|_{L^{2}\left(B_{n}\right)}^{2}\right) \leq 2 \int_{B_{n}} b \partial_{t} u_{n} . \partial_{x}^{2} u_{n} d t d x+K_{\epsilon}\|f\|_{L^{2}(B)}^{2} .
$$


Let us now consider the term $2 \int_{B_{n}} b \partial_{t} u_{n} . \partial_{x}^{2} u_{n} d t d x$. We have

$$
\begin{aligned}
2 \int_{B_{n}} b \partial_{t} u_{n} \cdot \partial_{x}^{2} u_{n} d t d x & =2 \int_{B_{n}}\left(b \partial_{x}\left(\partial_{t} u_{n} . \partial_{x} u_{n}\right) d t d x+b \partial_{t}\left(\partial_{x} u_{n}\right)^{2}\right) d t d x \\
& =-\int_{0}^{1} b\left(\partial_{x} u_{n}(n, x)\right)^{2} d x+2 \int_{B_{n}} b^{\prime}\left(\partial_{x} u_{n}\right)^{2} d t . d x
\end{aligned}
$$

Note that the functions $b$ (which is positive) and $b^{\prime}$, defined by

$$
b^{\prime}(t)=\frac{c^{\prime}(t)}{\left(\varphi_{2}(t)-\varphi_{1}(t)\right)^{2}}-\frac{2 c(t)\left(\varphi_{2}^{\prime}(t)-\varphi_{1}^{\prime}(t)\right)}{\left(\varphi_{2}(t)-\varphi_{1}(t)\right)^{3}},
$$

are bounded by virtue of hypothesis $\left(H_{2}\right)$. Using Lemma 1 , this yields

$$
\begin{aligned}
2 \int_{B_{n}} b \partial_{t} u_{n} \cdot \partial_{x}^{2} u_{n} d t d x & \leq 2 \int_{B_{n}} b^{\prime}\left(\partial_{x} u_{n}\right)^{2} d t \cdot d x \\
& \leq K_{2}\left\|\partial_{x} u_{n}\right\|^{2} \\
& \leq K_{3}\|f\|^{2}
\end{aligned}
$$

where $\left(K_{i}\right)_{i=1,2}$ stand for constants independent of $n$. Consequently, choosing $\epsilon=1 / 2$ in the relationship (3.1) we obtain, thanks to condition (ii) of $\left(\mathrm{H}_{2}\right)$,

$$
\left\|\partial_{t} u_{n}\right\|^{2}+\left\|\partial_{x}^{2} u_{n}\right\|^{2} \leq K\|f\|^{2} .
$$

THEOREM 2. Suppose that the conditions $\left(H_{2}\right)$ are satisfied. Then, problem $\left(P_{2}\right)$ admits a (unique) solution $u \in H^{1,2}\left(D_{2}\right)$.

PROOF. We obtain the solution $u$ by letting $n$ go to infinity in the previous proposition. The uniqueness can be proven as in Theorem 1 .

\section{The case of a bounded triangular domain}

Let us consider the problem

$$
\left\{\begin{array}{l}
\partial_{t} u-c(t) \partial_{x}^{2} u=f \in L^{2}\left(D_{3}\right) \\
u_{\left.\mid \partial D_{3} \backslash\{T\} \times\right] \varphi_{1}(T), \varphi_{2}(T)[=0}
\end{array}\right.
$$

where

$$
D_{3}=\left\{(t, x) \in \mathbb{R}^{2} \mid 0<t<T, \varphi_{1}(t)<x<\varphi_{2}(t)\right\}
$$

and let $\left(H_{3}\right)$ denote the following conditions on the functions $\left(\varphi_{i}\right)_{i=1,2}$ and $c$ : 
(i) $\quad\left(\varphi_{i}\right)_{i=1,2}$ and $c$ are continuous functions on $[0, T]$, differentiable on $] 0, T[$ such that $\left|\varphi_{i}^{\prime}\right|\left(\varphi_{2}-\varphi_{1}\right) \leq \epsilon$ where $\epsilon$ is small enough;

(ii) $c(t)>0$, for all $t \in[0, T]$;

(iii) $\varphi_{1}(0)=\varphi_{2}(0)$;

(iv) $T<+\infty$, and $T$ is small enough.

Set

$$
\Omega_{n}=\left\{(t, x) \in D_{3} \mid \frac{1}{n}<t<T, \varphi_{1}(t)<x<\varphi_{2}(t)\right\} .
$$

Let $f$ be an element of $L^{2}\left(D_{3}\right)$. For all $n \in \mathbb{N}$, we set $f_{n}=f_{\mid \Omega_{n}}$. Theorem 1 gives the existence of a function $u_{n} \in H^{1,2}\left(\Omega_{n}\right)$ which is a solution of the problem

$$
\left\{\begin{array}{l}
\partial_{t} u_{n}-c(t) \partial_{x}^{2} u_{n}=f_{n} \in L^{2}\left(\Omega_{n}\right) \\
u_{\left.n \mid \partial \Omega_{n} \backslash\{T\} \times\right] \varphi_{1}(T), \varphi_{2}(T)[}=0 .
\end{array}\right.
$$

LEMMA 2. There exists a constant $K$ independent of $n$ such that for all $t \in] 0, T[$ :

(1) $\left\|u_{n}\right\|_{L^{2}\left(\Omega_{n}\right)} \leq K\left\|\left(\varphi_{2}-\varphi_{1}\right) \partial_{x} u_{n}\right\|_{L^{2}\left(\Omega_{n}\right)}$;

(2) $\int_{\varphi_{1}(t)}^{\varphi_{2}(t)} u_{n}^{2}(t, x) d x \leq K\left(\varphi_{2}-\varphi_{1}\right)^{4} \int_{\varphi_{1}(t)}^{\varphi_{2}(t)}\left(\partial_{x}^{2} u_{n}\right)^{2}(t, x) d x$;

(3) $\int_{\varphi_{1}(t)}^{\varphi_{2}(t)}\left(\partial_{x} u_{n}\right)^{2}(t, x) d x \leq K\left(\varphi_{2}-\varphi_{1}\right)^{2} \int_{\varphi_{1}(t)}^{\varphi_{2}(t)}\left(\partial_{x}^{2} u_{n}\right)^{2}(t, x) d x$;

(4) $\left\|\partial_{x} u_{n}\right\|_{L^{2}\left(\Omega_{n}\right)} \leq K\|f\|_{L^{2}\left(D_{3}\right)}$.

PROOF. (1) Inequality is a consequence of the Poincaré inequality.

The operator

$$
\begin{aligned}
H^{2}(0,1) \cap H_{0}^{1}(0,1) & \rightarrow L^{2}(0,1) \\
v & \rightarrow v^{\prime \prime},
\end{aligned}
$$

is an isomorphism. So, there exists a constant $K$ such that

$$
\left\{\begin{array}{l}
\|v\|_{L^{2}(0,1)} \leq K\left\|v^{\prime \prime}\right\|_{L^{2}(0,1)} \\
\left\|v^{\prime}\right\|_{L^{2}(0,1)} \leq K\left\|v^{\prime \prime}\right\|_{L^{2}(0,1)}
\end{array} .\right.
$$

The change of variables (for fixed $t) x$ in $y=(1-x) \varphi_{1}(t)+x \varphi_{2}(t)$ transforming the interval $(0,1)$ into the interval $\left(\varphi_{1}(t), \varphi_{2}(t)\right)$ leads to the estimates $(2)$ and (3).

To prove (4), it is sufficient to expand the scalar product $\left(f_{n}, u_{n}\right)$ and use the inequality (1) Indeed, we deduce, for all $\epsilon>0$,

$$
\begin{aligned}
\int_{B_{n}} c(t)\left(\partial_{x} u_{n}\right)^{2}(t, x) & \leq\left|\left(f_{n}, u_{n}\right)\right| \\
& \leq \frac{1}{\epsilon}\left\|f_{n}\right\|^{2}+\epsilon\left\|u_{n}\right\|^{2} \\
& \leq \frac{1}{\epsilon}\|f\|_{L^{2}\left(D_{3}\right)}^{2}+\epsilon K\left\|\left(\varphi_{2}-\varphi_{1}\right) \partial_{x} u_{n}\right\|_{L^{2}\left(\Omega_{n}\right)}^{2}
\end{aligned}
$$

However, $\varphi_{2}-\varphi_{1}$ is bounded and $c>\alpha$ according to the condition (ii) of $\left(H_{3}\right)$. Choosing $\epsilon$ small enough yields the desired result. 
PROPOSITION 2. There exists a constant $K$ independent of $n$ such that

$$
\left\|u_{n}\right\|_{H^{1,2}\left(\Omega_{n}\right)} \leq K\|f\|_{L^{2}\left(D_{3}\right)} .
$$

Proof. We have

$$
\left\|\partial_{t} u_{n}\right\|_{L^{2}\left(\Omega_{n}\right)}^{2}+\left\|c \partial_{x}^{2} u_{n}\right\|_{L^{2}\left(\Omega_{n}\right)}^{2}-2 \int_{\Omega_{n}} c(t) \partial_{t} u_{n} \cdot \partial_{x}^{2} u_{n} d t d x=\left\|f_{n}\right\|_{L^{2}\left(\Omega_{n}\right)}^{2},
$$

and, thanks to the relationship $\partial_{t} u_{n}+\varphi_{i}^{\prime}(t)\left(\partial_{x} u_{n}\right)=0$ on the boundary $\partial \Omega_{n}$, we show that

$$
\begin{aligned}
-2 & \int_{\Omega_{n}} c(t) \partial_{t} u_{n} \cdot \partial_{x}^{2} u_{n} d t d x \\
= & 2 \int_{\partial \Omega_{n}} c(t) \partial_{t} u_{n} \cdot \partial_{x} u_{n} d t+\int_{\partial \Omega_{n}} c(t)\left(\partial_{x} u_{n}\right)^{2} d x \\
& -\int_{\Omega_{n}} c^{\prime}(t)\left(\partial_{x} u_{n}\right)^{2} d t d x \\
= & -\int_{1 / n}^{T} 2 c(t) \varphi_{1}^{\prime}(t)\left(\partial_{x} u_{n}\right)^{2} d t+\int_{1 / n}^{T} 2 c(t) \varphi_{2}^{\prime}(t)\left(\partial_{x} u_{n}\right)^{2} d t \\
& +\int_{1 / n}^{T} c(t) \varphi_{1}^{\prime}(t)\left(\partial_{x} u_{n}\right)^{2} d t-\int_{1 / n}^{T} c(t) \varphi_{2}^{\prime}(t)\left(\partial_{x} u_{n}\right)^{2} d t \\
& -\int_{\Omega_{n}} c^{\prime}(t)\left(\partial_{x} u_{n}\right)^{2} d t d x \\
= & -\int_{1 / n}^{T} c(t) \varphi_{1}^{\prime}(t)\left(\partial_{x} u_{n}\right)^{2} d t+\int_{1 / n}^{T} c(t) \varphi_{2}^{\prime}(t)\left(\partial_{x} u_{n}\right)^{2} d t \\
& -\int_{\Omega_{n}} c^{\prime}(t)\left(\partial_{x} u_{n}\right)^{2} d t d x .
\end{aligned}
$$

So, since $c^{\prime}$ is bounded, Assertion (4) of Lemma 2 yields

$$
\begin{aligned}
& \left|-2 \int_{\Omega_{n}} c(t) \partial_{t} u_{n} \cdot \partial_{x}^{2} u_{n} d t d x\right| \\
& \quad \leq\left|\int_{1 / n}^{T} c(t) \varphi_{1}^{\prime}(t)\left(\partial_{x} u_{n}\right)^{2} d t\right|+\left|\int_{1 / n}^{T} c(t) \varphi_{2}^{\prime}(t)\left(\partial_{x} u_{n}\right)^{2} d t\right|+K\|f\|_{L^{2}\left(D_{3}\right)}^{2} .
\end{aligned}
$$

Now, we estimate the term $I=\left|\int_{1 / n}^{T} c(t) \varphi_{1}^{\prime}(t)\left(\partial_{x} u_{n}\right)^{2} d t\right|$. For this purpose, we set

$$
\psi(t, x)=\frac{\varphi_{2}(t)-x}{\varphi_{2}(t)-\varphi_{1}(t)}
$$


Hence,

$$
\begin{aligned}
I= & \int_{1 / n}^{T} c(t) \varphi_{1}^{\prime}(t)\left\{\int_{\varphi_{1}(t)}^{\varphi_{2}(t)} \partial_{x}\left[\psi(t, x)\left(\partial_{x} u_{n}(t, x)\right)^{2}\right] d x\right\} d t \\
= & \int_{\Omega_{n}} c(t) \varphi_{1}^{\prime}(t) \partial_{x}\left[\psi(t, x)\left(\partial_{x} u_{n}(t, x)\right)^{2}\right] d x d t \\
= & \int_{\Omega_{n}} \frac{c(t) \varphi_{1}^{\prime}(t)}{\varphi_{2}(t)-\varphi_{1}(t)}\left(\partial_{x} u_{n}(t, x)\right)^{2} d x d t \\
& \left.+2 \int_{\Omega_{n}} c(t) \varphi_{1}^{\prime}(t) \psi(t, x)\right) \partial_{x} u_{n}(t, x) \partial_{x}^{2} u_{n}(t, x) d x d t
\end{aligned}
$$

Note that there exists a constant $K$ such that

$$
\begin{aligned}
& \left|2 \int_{\Omega_{n}} c(t) \varphi_{1}^{\prime}(t) \psi(t, x) \partial_{x} u_{n}(t, x) \partial_{x}^{2} u(t, x) d x d t\right| \\
& \quad \leq K\left\|\partial_{x}^{2} u_{n}\right\|\left\|\varphi_{1}^{\prime} \partial_{x} u_{n}\right\| \\
& \quad \leq K \epsilon\left\|\partial_{x}^{2} u_{n}\right\| .
\end{aligned}
$$

(where $\epsilon=\sup \varphi_{1}^{\prime}\left(\varphi_{2}-\varphi_{1}\right)$ ). Furthermore,

$$
\begin{aligned}
& \left|\int_{\Omega_{n}} \frac{c(t) \varphi_{1}^{\prime}(t)}{\varphi_{2}(t)-\varphi_{1}(t)}\left(\partial_{x} u_{n}(t, x)\right)^{2} d x d t\right| \\
& \quad \leq K \int_{1 / n}^{T} \frac{c(t) \varphi_{1}^{\prime}(t)}{\varphi_{2}(t)-\varphi_{1}(t)}\left(\varphi_{2}(t)-\varphi_{1}(t)\right)^{2} \int_{\varphi_{1}(t)}^{\varphi_{2}(t)}\left(\partial_{x}^{2} u_{n}(t, x)\right)^{2} d x d t \\
& \quad \leq K \int_{\Omega_{n}} c(t) \varphi_{1}^{\prime}(t)\left(\varphi_{2}(t)-\varphi_{1}(t)\right)\left(\partial_{x}^{2} u_{n}(t, x)\right)^{2} d x d t \\
& \quad \leq K \epsilon\left\|\partial_{x}^{2} u_{n}\right\|^{2}
\end{aligned}
$$

Then, there exists a constant $K^{\prime}$ such that

$$
\left\|\partial_{t} u_{n}\right\|+\left\|\partial_{x}^{2} u_{n}\right\| \leq K^{\prime}\|f\| .
$$

Consequently,

$$
\left\|u_{n}\right\|_{H^{1,2}\left(\Omega_{n}\right)} \leq K^{\prime}\|f\| .
$$

THEOREM 3. Suppose that conditions $\left(H_{3}\right)$ are satisfied. Then, problem $\left(P_{3}\right)$ admits a (unique) solution $u \in H^{1,2}\left(D_{3}\right)$.

PROOF. Thanks to Proposition 2, the solution $u$ can be obtained by letting $n$ go to infinity. 


\section{The case of a sectorial domain}

In this section, we consider the problem

$$
\left\{\begin{array}{l}
\partial_{t} u-c(t) \partial_{x}^{2} u=f \in L^{2}\left(D_{4}\right) \\
u_{\mid \partial D_{4}}=0
\end{array}\right.
$$

where

$$
D_{4}=\left\{(t, x) \in \mathbb{R}^{2} \mid 0<t<+\infty, \varphi_{1}(t)<x<\varphi_{2}(t)\right\}
$$

under the hypotheses $\left(H_{4}\right)$ on the functions $\left(\varphi_{i}\right)_{i=1,2}$ and $c$ :

(i) $\left\{\begin{array}{l}\left(\varphi_{i}\right)_{i=1,2} \text { and } c \text { are continuous functions on }[0,+\infty[\text {, differentiable on } \\ ] 0,+\infty\left[\text {; here }\left|\varphi_{i}^{\prime}\right|\left(\varphi_{2}-\varphi_{1}\right) \text { is small enough in a neighborhood of } 0 \text { and }\right. \\ \left(\varphi_{i}^{\prime}\right)_{i=1,2} \text { is bounded in a neighborhood of }+\infty .\end{array}\right.$

(ii) $\varphi_{2}-\varphi_{1}$ is increasing a neighborhood of $+\infty$ or

$$
\text { there exists } M>0, \quad\left|\varphi_{1}^{\prime}(t)-\varphi_{2}^{\prime}(t)\right|\left(\varphi_{2}(t)-\varphi_{1}(t)\right) \leq M . c(t) \text {; }
$$

(iii) there exist $\alpha_{i}>0, i=1,2$ such that $\alpha_{1} \geq c(t) \geq \alpha_{2}>0$, for all $t \in[0,+\infty[$;

(iv) $\varphi_{1}(0)=\varphi_{2}(0)$;

(v) $T=+\infty$.

In order to prove our main result, we need the following trace theorem [15, Theorem 2.1, Chapter 4]:

\section{THEOREM 4.}

(i) If $u \in H^{1,2}(] 0, T[\times] 0,1[)$, then

$$
u_{\mid\{0\} \times] 0,1[} \in H_{0}^{1}(0,1)=\left\{u \in H^{1}(0,1) \mid u(0)=u(1)=0\right\} .
$$

(ii) If $\varphi \in H_{0}^{1}(0,1)$, there exists $u \in H^{1,2}(] 0, T[\times] 0,1[)$ such that $u_{\{0\} \times] 0,1[}=\varphi$ and $u_{\mid] 0, T[\times\{0\} \cup] 0, T[\times\{1\}}=0$.

Corollary 1. Let $\varphi$ be an element of $H_{0}^{1}(0,1)$. If hypotheses $\left(H_{1}\right)$ are fulfilled, then the problem

$$
\left\{\begin{array}{l}
\partial_{t} u-c(t) \partial_{x}^{2} u=f \in L^{2}\left(D_{1}\right) \\
u_{\left.\right|_{\{0\} \times] \varphi_{1}(0), \varphi_{2}(0)[}=\varphi}=\varphi \\
u_{\left.\right|_{\left.\partial D_{1} \backslash\{0\} \times\right] \varphi_{1}(0), \varphi_{2}(0)[\cup\{T\} \times] \varphi_{1}(T), \varphi_{2}(T)[}=0,}=0,
\end{array}\right.
$$

admits a solution $u \in H^{1,2}\left(D_{1}\right)$.

THEOREM 5. Suppose that the conditions $\left(H_{4}\right)$ are satisfied. Then, problem $\left(P_{4}\right)$ admits a (unique) solution $u \in H^{1,2}\left(D_{4}\right)$. 
PROOF. The proof of this result can be obtained by 'subdividing' the domain $D_{4}$ in three open subdomains $D_{1}, D_{2}$ and $D_{3}$ which respectively verify the hypotheses $\left(H_{1}\right)$, $\left(H_{2}\right)$ and $\left(H_{3}\right)$. Furthermore, we impose $\overline{D_{4}}=\bigcup_{i=1,2,3} \overline{D_{i}}$. This is possible thanks to $\left(\mathrm{H}_{4}\right)$.

Corollary 1 allows us to solve the problem posed in every subdomain $\left(D_{i}\right)_{i=1,2,3}$, and obtain solutions $u_{1}, u_{2}$ and $u_{3}$ respectively in $D_{1}, D_{2}$ and $D_{3}$ which coincide on the common segments of $\left(\overline{D_{i}}\right)_{i=1,2,3}$, that is, $u_{1}=u_{2}$ on $\overline{D_{1}} \cap \overline{D_{2}}$ and $u_{2}=u_{3}$ on $\overline{D_{2}} \cap \overline{D_{3}}$. The solution $u$ in $D_{4}$ is then defined by $u_{\mid D_{i}}=u_{i}$ for all $i=1,2,3$.

\section{REMARK 2.}

(1) In the case where $\varphi_{1}=0$ and $\varphi_{2}(t)=t^{\alpha}$, it is easy to see that the condition $\alpha>1 / 2$ satisfies hypothesis $\left(H_{4}\right)$.

(2) This work may be extended to other operators (with constant or variable coefficients). Moreover, we can consider the case where the second member is more regular or lies in non-Hilbertian Sobolev spaces (built on Lebesgue spaces $\left.L^{p}\right)$.

(3) Instead of looking for the boundary conditions assuring the existence of the solution in the natural space, we can choose a 'bad' domain which generates some singularities in the solution. Then, the following two questions arise.

(a) What is the optimal regularity of this singular part?

(b) What is the number of the singularities which generate the singular part?

\section{References}

[1] Yu. A. Alkhutov and I. T. Mamedov, 'The first boundary value problem for nondivergence secondorder parabolic equations with discontinuous coefficients', Russian Acad. Sc. Sbornik Math. 59(2) (1988), 471-495.

[2] A. Azzam, 'On behavior of solution of elliptic and parabolic equations at a crack', Hokkaido Math. J. 19(2) (1990), 339-344.

[3] E. A. Baderko, 'Étude du problème de Dirichlet pour une équation $2 p$-parabolique dans un domaine non rectangulaire', C. R. Math. Acad. Sci. Paris 287 (1978), 221-224.

[4] J. Cea et al. (ed.), Partial Differential Equations and Functional Analysis in Memory of Pierre Grisvard (Birkhäuser, Basel, 1996).

[5] M. Dauge, Elliptic Boundary Value Problems on Corner Domains (Springer, Berlin, 1988).

[6] P. Grisvard, Elliptic Problems in Non-smooth Domains, Monographs and Studies in Mathematics, 24 (Pitman, Boston, MA, 1985).

[7] P. Grisvard, Singularities in Boundary Value Problems, RMA, 22 (Masson, Paris, 1992).

[8] P. Grisvard, Problèmes aux limites dans des domaines avec points de rebroussement, in: Partial Differential Equations and Functional Analysis in Memory of P. Grisvard (Birkhäuser, Basel, 1996), pp. 1-17.

[9] K. Ibuki, 'On the regularity of a mixed problem for hyperbolic equation of second order in a domain with corners', J. Math. Kyoto Univ. 16(1) (1976), 167-183.

[10] V. A. Kondratiev, 'Boundary-value problems for elliptic equations in domains with conical or angular points', Trans. Moscow Math. Soc. 16 (1967), 227-313.

[11] R. Labbas and M. Moussaoui, 'On the resolution of the heat equation with discontinuous coefficients', Semigroup Forum 60 (2000), 187-201. 
[12] R. Labbas and B.-K. Sadallah, 'Smoothness of the solution of a fourth order parabolic equation in a polygonal domain', Int. J. Appl. Math. 1(1) (1999), 75-90.

[13] R. Labbas, A. Medeghri and B.-K. Sadallah, 'On a parabolic equation in a triangular domain', Appl. Math. Comput. 130(2-3) (2002), 511-523.

[14] R. Labbas, A. Medeghri and B.-K. Sadallah, 'Sur une équation parabolique dans un domaine non cylindrique', C. R. Math. Acad. Sci. Paris 335(12) (2002), 1017-1022.

[15] J.-L. Lions and E. Magenes, Problèmes aux limites non homogènes et applications, Vol. 2 (Dunod, Paris, 1968).

[16] V. G. Maz'ja and B. A. Plamenevskii, 'Weighed spaces with nonhomogeneous norms and boundary value problems in domains with conical points', Amer. Math. Soc. Transl. 2(123) (1984), 89-107.

[17] M. Moussaoui and B.-K. Sadallah, 'Régularité des coefficients de propagation de singularités pour l'Equation de la Chaleur dans un Ouvert Plan polygonal', C. R. Math. Acad. Sci. Paris 293(5) (1981), 297-300.

[18] S. Nicaise, 'Regularity of the solutions of elliptic systems in polyhedral domains', Bull. Belg. Math. Soc. Simon Stevin 4 (1997), 411-429.

[19] B.-K. Sadallah, 'Étude d'un problème $2 m$-parabolique dans des domaines plans non rectangulaires', Boll. U. M. I. B. (6) 2(1) (1983), 51-112.

[20] M. Taniguchi, 'Initial boundary value problem for the wave equation in a domain with a corner', Tokyo J. Math. 16(1) (1993), 61-98.

BOUBAKER-KHALED SADALLAH, Laboratoire Equations aux Dérivées Partialles et Histoire des Mathématiques, Department of Mathematics, École Normale Supérieure, 16050-Kouba, Algiers, Algeria

e-mail: sadallah@ens-kouba.dz 